\section{Phase I/II trial of cladribine, high-dose cytarabine, mitoxantrone, and G-CSF with dose-escalated mitoxantrone for relapsed/refractory acute myeloid leukemia and other high-grade myeloid neoplasms}

Current treatment results for relapsed/refractory acute myeloid leukaemia (AML) are typically poor. ${ }^{1}$ Because some data suggest the value of increased anthracycline doses with intensive AML chemotherapies, ${ }^{2,3}$ we conducted a Phase I/II trial (ClinicalTrials.gov: NCT02044796) to test the efficacy of treatment with escalated doses of mitoxantrone in association with CLAG-M (or 'GCLAM'; cladribine/high-dose cytarabine/mitoxantrone/G-CSF) in adults $\geq 18$ years with relapsed or refractory AML and other high-grade myeloid neoplasms $(\geq 10 \%$ myeloblasts in blood and/or marrow).

Patients were eligible if they had a treatment-related mortality score of $\leq 6.9$ (corresponding to a $\leq 6.9 \%$ probability of 4-week mortality ${ }^{4}$. While this score was developed for adults with newly-diagnosed AML - and thus does not account for some variables relevant for relapsed/refractory disease ${ }^{5}$ - we have previously used it in the relapsed/refractory disease setting. ${ }^{6,7}$ Patients were also required to have a left ventricular ejection fraction $\geq 45 \%$, creatinine $\leq 2.0 \mathrm{mg} / \mathrm{dL}$, and bilirubin $\leq 2.5$ times the upper limit of normal, no uncontrolled infection, and expected survival of $>1$ year absent AML; for details, see Online Supplementary Information. In phase I, groups of 612 patients received $12,14,16$, or $18 \mathrm{mg} / \mathrm{m}^{2}$ (dose levels 1 - 4) of intravenous (IV) mitoxantrone on days 1-3. Granulocyte colony-stimulating factor (G-CSF) was given subcutaneously (days $0-5$ ), cladribine IV at 5 $\mathrm{mg} / \mathrm{m}^{2}$ (days 1-5), and cytarabine IV at $2 \mathrm{~g} / \mathrm{m}^{2}$ (days 1-5). The first 2 doses of G-CSF could be omitted if the total white blood cell count was $>20,000 / \mu \mathrm{L}$. Patients in phase II received mitoxantrone at the recommended phase II dose (RP2D) determined in phase I. A second, identical cycle of CLAG-M was given to patients who did not achieve complete remission (CR) or CR with incomplete hematologic recovery (CRi) in the first cycle. Patients in CR/CRi after one to two cycles could then receive up to 4 cycles of CLAG (mitoxantrone omitted). The protocol was approved by our Institutional Review Board, and patients gave written informed consent in accordance with the Declaration of Helsinki.

Between 2/2014 and 4/2017, we enrolled 60 patients (Table 1). Among the 26 patients enrolled in phase I (Online Supplementary Table S1), one dose-limiting toxicity occurred at dose level $1\left(12 \mathrm{mg} / \mathrm{m}^{2}\right.$ : nausea) and two at dose level $4\left(18 \mathrm{mg} / \mathrm{m}^{2}\right.$ : encephalopathy and cardiogenic shock). Online Supplementary Table S2 summarizes phase I treatment outcomes, establishing CLAG-M with mitoxantrone at $16 \mathrm{mg} / \mathrm{m}^{2}$ as the maximum tolerated dose/RP2D. This contrasts with our experience in a parallel cohort of adults with newly-diagnosed disease, in which all tested dose levels of mitoxantrone (including $18 \mathrm{mg} / \mathrm{m} 2$ ) were well tolerated. ${ }^{8}$ Whether this difference reflects a greater vulnerability of patients with relapsed/refractory disease to higher doses of mitoxantrone or whether it is a chance finding due to the small patient numbers is unknown.

Forty patients (including 6 in phase I) with a median age of 63 [range: 33-77] years were treated at the RP2D (Table 1). Table 2 summarizes best responses after 1-2 treatment cycles for the entire study population and for those treated at the RP2D. CR or CRi were achieved in
11 (28\% [95\% confidence interval: 15-44\%]) and 13 (32\% [19-49\%]) patients, giving a CR/CRi rate of $60 \%$ $(43-75 \%)$. Two of the 10 patients who received a second cycle of therapy had an improvement in response. Nine of the $11 \mathrm{CR}(82 \%)$ and $10 / 13$ of the CRi $(77 \%)$ were negative for measurable residual disease $\left(\mathrm{MRD}^{\text {neg }}\right)$ as determined by flow cytometry. Among the others, 1 patient achieved a morphologic leukaemia-free state and 11 had resistant disease; two additional patients had normalized blood counts after CLAG-M but bone marrow examinations were not performed, limiting response assessment. Four- and 8-week mortality was $5 \%$ (2 deaths in aplasia). Infections and neutropenic fever were the most common non-hematologic grade 3-

Table 1. Characteristics of the study cohort.

\begin{tabular}{|c|c|c|}
\hline Parameter & $\begin{array}{l}\text { Total Study } \\
\text { Cohort, } n=60\end{array}$ & $\begin{array}{c}\text { RP2D Cohort, } \\
n=40\end{array}$ \\
\hline Median age (range), years & $61(33-77)$ & $63(33-77)$ \\
\hline Male gender, n (\%) & $35(58 \%)$ & $24(60 \%)$ \\
\hline $\begin{array}{l}\text { Disease type } \\
\text { AML } \\
\text { With recurrent genetic abnormalities } \\
\text { With mutated NPM1 } \\
\text { With biallelic CEBPA mutation } \\
\text { With myelodysplasia-related changes } \\
\text { Treatment-related AML } \\
\text { AML from antecedent hematologic disorde } \\
\text { AML, not otherwise specified } \\
\text { MDS-EB2 }\end{array}$ & $\begin{array}{c}3(5 \%) \\
8(13 \%) \\
1(2 \%) \\
11(18 \%) \\
5(8 \%) \\
\text { er } 11(18 \%) \\
14(23 \%) \\
7(12 \%)\end{array}$ & $\begin{array}{c}2(5 \%) \\
6(15 \%) \\
1(2 \%) \\
7(18 \%) \\
3(8 \%) \\
7(18 \%) \\
8(20 \%) \\
6(15 \%)\end{array}$ \\
\hline Secondary disease* & $16(27 \%)$ & $10(25 \%)$ \\
\hline $\begin{array}{l}\text { Disease status, } \mathrm{n}(\%) \\
\text { Primary refractory } \\
\text { Relapse } \\
\text { CR1 duration, months: median (range) } \\
\text { Prior HCT } \\
\text { Number of prior therapies median (range) }\end{array}$ & $\begin{array}{c}25(42 \%) \\
35(58 \%) \\
9(1-120) \\
13(22 \%) \\
2(1-6)\end{array}$ & $\begin{array}{c}20(50 \%) \\
20(50 \%) \\
11.5(1-86) \\
7(18 \%) \\
2(1-6)\end{array}$ \\
\hline Median TRM score (range) & $1.99(0.25-6.39)$ & $2.10(0.25-6.39)$ \\
\hline $\begin{array}{l}\text { Performance status, n (\%) } \\
0 \\
1 \\
2\end{array}$ & $\begin{array}{c}19(32 \%) \\
39(65 \%) \\
2(3 \%)\end{array}$ & $\begin{array}{c}9(23 \%) \\
29(72 \%) \\
2(5 \%)\end{array}$ \\
\hline $\begin{array}{l}\text { Cytogenetic risk, } n(\%) \\
\text { Intermediate } \\
\text { Adverse }\end{array}$ & $\begin{array}{l}36(60 \%) \\
24(40 \%)\end{array}$ & $\begin{array}{l}25(62 \%) \\
15(38 \%)\end{array}$ \\
\hline
\end{tabular}

Mutational status, n (\%)

FLT3-internal tandem duplication

Wild-type

Mutated

$33(55 \%)$

$4(7 \%)$

$23(38 \%)$

$25(42 \%)$

Unknown

$23(38 \%)$

Wild-type

Mutated

$1(2 \%)$

$17(42 \%)$

$8(13 \%)$

$27(45 \%)$

Unknown$$
\text { ge) }
$$

WBC (x 10)

Peripheral blood blasts (\%)

Hemoglobin ( $\mathrm{g} / \mathrm{dL}$ )

Platelets (x $\left.10^{\circ} \mathrm{L}\right)$

Creatinine $(\mathrm{mg} / \mathrm{dL})$

Total bilirubin (mg/dL)

$3(0-51)$
$4(0-84)$
$10.0(6.8-14.9)$
$66(6-328)$
$.86(0.47-1.63)$
$0.6(0.2-2.0)$

$14(35 \%)$

$6(15 \%)$

$20(50 \%)$

* Defined either as AML transformed from antecedent hematologic disorder, or AML/MDS in a patient who had previously received cytotoxic therapy; RP2D: recommended phase II dose; CR1: duration of first complete remission; HCT: hematopoietic stem cell transplant; TRM: treatmentrelated mortality 
5 toxicities (Online Supplementary Table S3). Comparisons between patients with primary refractory $v s$. relapsed disease revealed no difference in safety, early mortality rates, adverse event rates, or rates of subsequent haematopoietic cell transplantation (HCT) (Online Supplementary Table S4). Among the CR patients, the median time to an absolute neutrophil count $\geq 1,000 / \mu \mathrm{L}$ was 29 (range: $27-67$ ) days; the median time to a platelet count $\geq 100,000 / \mu \mathrm{L}$ was also 29 (range: $22-67$ ) days.

Fourteen of the 24 responders were taken off the protocol to undergo HCT, and 8 received alternative consolidation chemotherapies, many prior to transplantation. Twenty-two $(55 \%)$ have received HCT to date, including $17 / 24(71 \%)$ of the responders. Relapse, including emergence of minimal residual disease, occurred in 11 patients, while 3 patients died in remission after CR durations of 128,167 , and 379 days. With a median follow up among living patients of 20 months, the median OS and RFS for the RP2D group were 11 and 12 months (Figures 1A and 1B), with 1-year OS and RFS estimates of $44 \%$ and $47 \%$.

We next compared response rates and tolerability of CLAG-M in younger and older participants (Online Supplementary Table S5). CR rates in those $<65$ years $v$.
Table 2. Best response after 1-2 cycles of study therapy.

\begin{tabular}{|c|c|c|}
\hline Response & $\begin{array}{c}\text { All patients } \\
(n=60)\end{array}$ & $\begin{array}{l}\text { Patients treated } \\
\text { at RP2D, }(n=40)\end{array}$ \\
\hline $\begin{array}{l}\mathrm{CR} \\
\mathrm{MRD}^{\text {neg }} \\
\mathrm{MRD}^{\text {pos }}\end{array}$ & $\begin{array}{l}18(30 \%) \\
12(20 \%) \\
6(10 \%)\end{array}$ & $\begin{array}{l}11(28 \%) \\
9(22 \%) \\
2(5 \%)\end{array}$ \\
\hline $\begin{array}{l}\text { CRi } \\
\text { MRD }^{\text {neg }} \\
\text { MRD }^{\text {pos }}\end{array}$ & $\begin{array}{c}16(27 \%) \\
13(22 \%) \\
3(5 \%)\end{array}$ & $\begin{array}{c}13(32 \%) \\
10(25 \%) \\
3(8 \%)\end{array}$ \\
\hline CR/CRi rate & $34(57 \%)$ & $24(60 \%)$ \\
\hline $\begin{array}{l}\text { Morphologic leukemia-free state } \\
\text { MRD } \\
\text { MRD }^{\text {nos }}\end{array}$ & $\begin{array}{l}3(5 \%) \\
2(3 \%) \\
1(2 \%)\end{array}$ & $\begin{array}{c}1(2 \%) \\
0 \\
1(2 \%)\end{array}$ \\
\hline Resistant disease & 17 (28\%) & $11(28 \%)$ \\
\hline Not evaluable* & $3(5 \%)$ & $2(5 \%)$ \\
\hline Death in aplasia^ & $3(5 \%)$ & $2(5 \%)$ \\
\hline
\end{tabular}

${ }^{+} 6$ patients treated at RP2D were part of the phase I cohort. ${ }^{*}$ One of these patients had normal count recovery but refused marrow assessment, one person had normal count recovery and went to comfort care due to infections, and one patient had partial count recovery (ANC $>500 / \mu L$, platelet count $>50,000 / \mu L$ ) but refused bone marrow restaging. $\wedge$ Death within 28 days of initiation of study therapy without evidence of disease; RP2D: recommended phase II dose; CR: complete remission; MRD: measurable residual disease; $C R i$ : $C R$ with incomplete hematologic recovery.
A

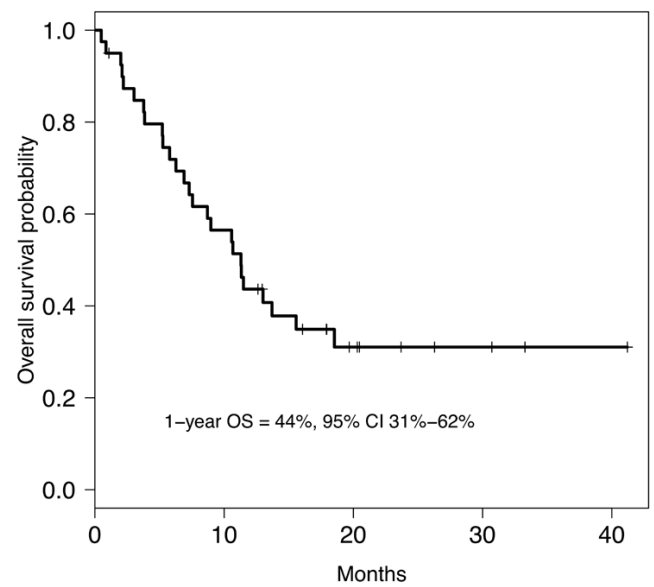

C

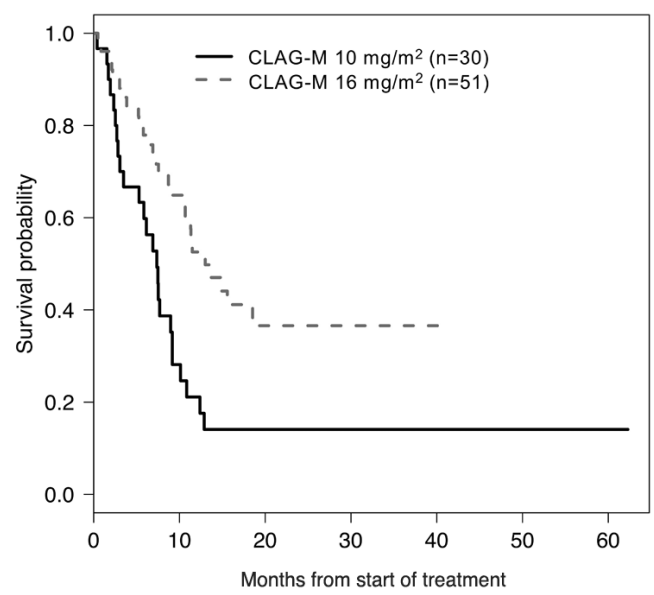

B

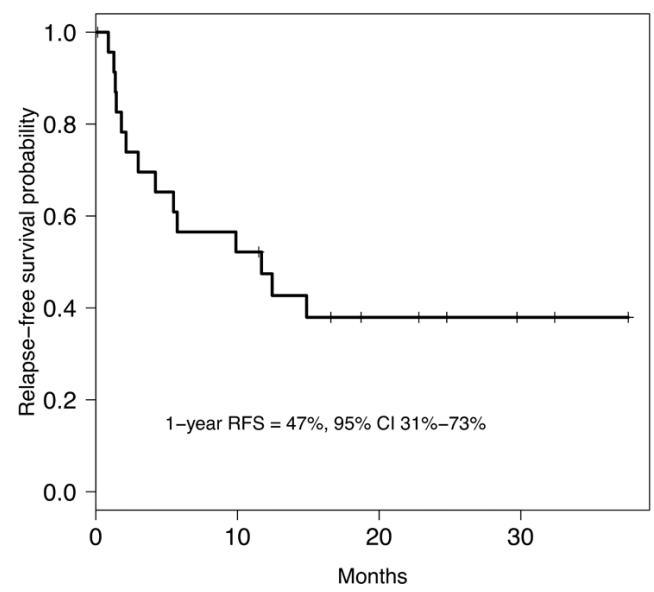

D

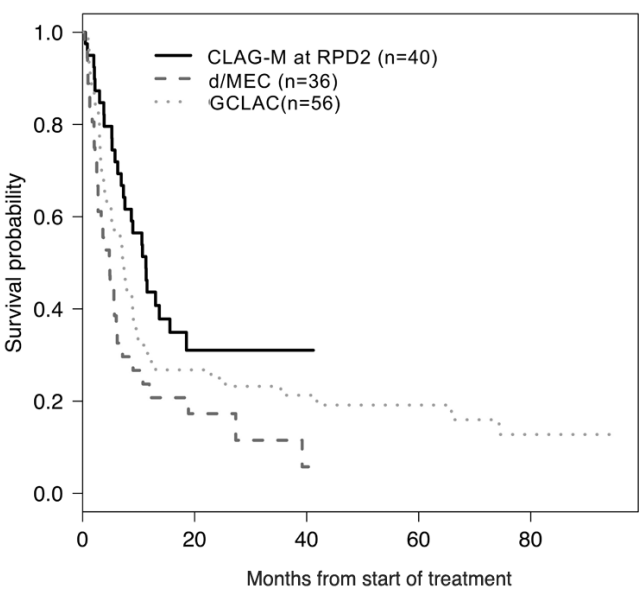

Figure 1. Survival estimates for patients treated with CLAG-M at the RP2D and other high-dose cytarabine-containing salvage regimens. Kaplan-Meier estimates of (A) overall survival and (B) relapse-free survival of the 40 patients who received CLAG-M at the RP2D (i.e., using mitoxantrone at a dose of $16 \mathrm{mg} / \mathrm{m}^{2}$ ). (C) Kaplan-Meier estimates of overall survival of TRM score-matched patients who received CLAG-M with mitoxantrone at $10 \mathrm{mg} / \mathrm{m}^{2}$ compared to patients who received CLAG-M with mitoxantrone at $16 \mathrm{mg} / \mathrm{m}^{2}$. (D) Kaplan-Meier estimates of overall survival of patients who received CLAG-M at the R2PD in our study (mitoxantrone at $16 \mathrm{mg} / \mathrm{m}^{2}$ ) compared to similar patients who received d/MEC and GCLAC at our institution. 
$\geq 65$ years were $32 \%$ vs. $22 \%, \mathrm{MRD}^{\text {neg }} \mathrm{CR}$ rates were $27 \%$ vs. $17 \%$, and CR/CRi rates were $64 \%$ vs. $56 \%$. Four- and 8-week mortality rates were low in both age groups $(5 \% / 5 \%$ and $6 \% / 6 \%$ in younger $v s$. older patients). Rates of subsequent allogeneic HCT were similar (55\% vs. $56 \%$, younger vs. older). Median OS was 13 months for younger and 11 months for older patients; 1 year OS were $52 \%$ and $33 \%$, respectively. Likewise, median RFS was 14 months for younger and 8 months for older patients (1-year RFS of $54 \%$ vs. $40 \%$ ).

To determine whether escalation of mitoxantrone increases the anti-leukemia efficacy of CLAG-M, we took advantage of the fact that since 2012, a larger number of adults at our institution have received CLAG-M with mitoxantrone at $10 \mathrm{mg} / \mathrm{m}^{2}$ (the dose reported originally by Polish investigators ${ }^{9}$ ) for relapsed/refractory AML and other high-grade myeloid neoplasms. In our comparisons, we included 51 patients treated with CLAG-M with mitoxantrone at $16 \mathrm{mg} / \mathrm{m}^{2}$ (40 on-study patients treated at the RP2D and 11 patients treated offprotocol) and 30 patients treated with CLAG-M with mitoxantrone at $10 \mathrm{mg} / \mathrm{m}^{2}$ (all off-study), who all met the medical criteria for enrollment in our phase I/II study (i.e., treatment-related mortality (TRM) score $\leq 6.9$, adequate organ function; see Online Supplementary Table S6 for patient characteristics). In multivariable analysis controlling for several prognostic factors, compared to patients treated with CLAG-M with mitoxantrone at 10 $\mathrm{mg} / \mathrm{m}^{2}$, CLAG-M with mitoxantrone at $16 \mathrm{mg} / \mathrm{m}^{2}$ was associated with significantly longer OS (hazard ratio $[\mathrm{HR}]=2.65$ [1.44-4.89], $P=0.0018$; Figure $1 \mathrm{C})$ and nonsignificantly longer RFS ( $\mathrm{HR}=1.51$ [0.62-3.69], $P=0.37)$, while remission rates were not different (Online Supplementary Table S7). Why the difference in outcomes was particularly noticeable for OS is unclear. The fact that a higher proportion of patients treated with mitoxantrone at $16 \mathrm{mg} / \mathrm{m}^{2}$ underwent subsequent allogeneic HCT may play a role. However, an additional multivariable analysis in which allogeneic HCT was included as a time-dependent covariate showed a qualitatively similar result (for OS: $H R=2.1, P=0.011$ ). We also performed analyses in which we included all institutional patients treated with CLAG-M with mitoxantrone at either 16 $\mathrm{mg} / \mathrm{m}^{2}(\mathrm{n}=55)$ or $10 \mathrm{mg} / \mathrm{m}^{2}(\mathrm{n}=43)$ regardless of TRM score and, controlling for TRM, obtained results qualitatively similar to those for patients with TRM score $\leq 6.9$ (Online Supplementary Figure $S 1$ and Online Supplementary Table S8).

Finally, we compared the outcomes of this study to those obtained with other high-dose cytarabine-containing salvage regimens used at our institution, namely GCLAC (G-CSF/clofarabine/cytarabine; $n=56$ ) and decitabine-primed MEC (d/MEC: decitabine/mitoxantrone/etoposide/cytarabine; $\mathrm{n}=36$ ), which were given as part of two phase I/II studies (NCT00602225, ${ }^{10,11}$ NCT017298457) or off-protocol, controlling for prognostic factors and restricting inclusion to patients with TRM scores of $\leq 6.9$ (see Online Supplementary Table S9 for characteristics). A higher proportion of CLAG-M patients underwent subsequent HCT $(55 \%)$ than did d/MEC or GCLAC patients $(29 \%$ and $45 \%$, respectively). At univariate analysis, patients treated with CLAG$M$ were more likely to have achieved CR/CRi than were patients on either of the other regimens. After controlling for prognostic factors by means of multivariable analysis (age, cytogenetic risk group, duration of CR1 and receipt of prior HCT), treatment with CLAG-M was associated with a higher likelihood of achieving CR/CRi than treatment with $\mathrm{d} / \mathrm{MEC}$ (odds ratio $[\mathrm{OR}]=0.35[0.13$ -
0.98], $P=0.05)$; but not than treatment with GCLAC $(\mathrm{OR}=0.55$ [0.21-1.43], $P=0.22$ (Online Supplementary Table S10). Cytogenetic risk was the only independent risk factor besides treatment. More importantly, patients treated with CLAG-M had statistically significantly longer OS than those treated with $\mathrm{d} / \mathrm{MEC}$ after multivariable adjustment $(\mathrm{HR}=2.02$ [1.15-3.53], $P=0.01)$, whereas there were no statistically significant differences in survival compared to GCLAC-treated patients $(\mathrm{HR}=1.46$ [0.86-2.49], $P=0.16$; Figure $1 \mathrm{D}$ and Online Supplementary Table S10). Additional multivariable analyses in which allogeneic HCT was included as timedependent covariate showed qualitatively similar results (for OS of CLAG-M vs. d/MEC: $\mathrm{HR}=2.04, P=0.008$; for OS of CLAG-M vs. GCLAC: $\mathrm{HR}=1.27, P=0.44)$.

In summary, CLAG-M with mitoxantrone up to 16 $\mathrm{mg} / \mathrm{m}^{2}$ appears safe and relatively well tolerated in fit younger and older adults with relapsed/refractory AML and other high-grade myeloid neoplasms. Escalating the mitoxantrone dose may provide additional antileukemia efficacy over the dose conventionally used with CLAG-M, although non-randomized comparisons and small cohort sizes limit the conclusions we can draw from our analyses. Acknowledging the same limitations, our analyses suggest that outcomes with CLAG-M with escalated mitoxantrone doses may compare favourably to those obtained with another high-dose cytarabinebased salvage regimen, $\mathrm{d} / \mathrm{MEC}$, and may be at least as good as with GCLAC. Our study thus adds to the evidence supporting the use of CLAG-M for intensive AML re-induction therapy, perhaps particularly with higher mitoxantrone doses.

Anna B. Halpern, ${ }^{1,2}$ Megan Othus, ${ }^{3}$ Emily M. Huebner, Bart L. Scott, ${ }^{1,4}$ Paul C. Hendrie, ${ }^{2,5}$ Mary-Elizabeth $M$. Percival, ${ }^{1,2}$ Pamela S. Becker, ${ }^{1,2}$ Heather A. Smith, ${ }^{5}$ Vivian G. Oehler, ${ }^{1,2}$ Johnnie J. Orozco, ${ }^{1,2}$ Ryan D. Cassaday, ${ }^{1,2}$ Kelda M. Gardner, ${ }^{2}$ Tara L. Chen, ${ }^{6}$ Sarah A. Buckley, Kaysey F. Orlowski, ${ }^{1}$ Asma Anwar, ${ }^{7}$ Elihu H. Estey ${ }^{1,2}$ and Roland B. Walter ${ }^{1,2,8}$

Clinical Research Division, Fred Hutchinson Cancer Research Center/University of Washington; 'Department of Medicine, Division of Hematology, University of Washington; ${ }^{3}$ Public Health Sciences Division, Fred Hutchinson Cancer Research Center; ${ }^{4}$ Department of Medicine, Division of Medical Oncology, University of Washington; 'Seattle Cancer Care Alliance; ${ }^{6}$ Deparment of Pharmacy Services, University of Washington, ${ }^{7}$ Hematology/Oncology Fellowship Program, University of Washington/Fred Hutchinson Cancer Research Center and ${ }^{8}$ Department of Epidemiology, University of Washington, Seattle, WA, USA

Acknowledgements: $A B H, S A B$, and $A A$ were supported by a fellowship training grant from the National Heart, Lung, and Blood Institute/National Institutes of Health (NHLBI/NIH:

T32-HL007093). The content is solely the responsibility of the authors and does not necessarily represent the official views of the $\mathrm{NIH}$. ABH is the recipient of a Conquer Cancer

Foundation/American Society of Clinical Oncology (ASCO) Young Investigator Award. RBW is a Leukemia \& Lymphoma Society Scholar in Clinical Research.

Correspondence: ROLAND B. WALTER -

rwalter@fredhutch.org

doi:10.3324/haematol.2018.204792

Information on authorship, contributions, and financial \& other disclosures was provided by the authors and is available with the online version of this article at www. haematologica.org. 
LETTERS TO THE EDITOR

References

1. Theol F, Schlenk RF, Heuser M, Gander A. How I treat refractory and early relapsed acute myeloid leukemia. Blood. 2015;126(3):319-327.

2. Fernandez HF, Sun Z, Yo X, et al. Anthracycline dose intensificatimon in acute myeloid leukemia. N Angl J Med. 2009;361(13):12491259.

3. Löwenberg B, Ossenkoppele GJ, van Putter W, et al. High-dose daunorubicin in older patients with acute myeloid leukemia. N Encl J Med. 2009;361(13):1235-1248.

4. Walter RB, Othus M, Borthakur G, et al. Prediction of early death after induction therapy for newly diagnosed acute myeloid leukemia with pretreatment risk scores: a novel paradigm for treatment assignment. J Chin Oncol. 2011;29(33):4417-4423.

5. Godwin CD, Othus M, Powell MA, Buckley SA, Ester EH, Walter RB. Prediction of early death in adults with relapsed or refractory acute myeloid leukemia. Leak Lymphoma. 2016;57(10):2421-2424.

6. Chen TL, Estey EH, Othus M, Gardner KM, Markle LJ, Walter RB. Cyclosporine modulation of multidrug resistance in combination with pravastatin, mitoxantrone and etoposide for adult patients with relapsed/refractory acute myeloid leukemia: a phase $1 / 2$ study. Leak Lymphoma. 2013;54(11):2534-2536.

7. Halpern AB, Othus M, Huebner EM, et al. Mitoxantrone, etopo- side and cytarabine following epigenetic priming with decitabine in adults with relapsed/refractory acute myeloid leukemia or other high-grade myeloid neoplasms: a phase $1 / 2$ study. Leukemia. 2017;31(12):2560-2567.

8. Halpern AB, Othus M, Huebner EM, et al. Phase $1 / 2$ trial of GCLAM with dose-escalated mitoxantrone for newly diagnosed AML or other high-grade myeloid neoplasms. Leukemia. 2018;32(11):2352-2362.

9. Wierzbowska A, Robak T, Plata A, et al. Cladribine combined with high doses of arabinoside cytosine, mitoxantrone, and GCSF (CLAG-M) is a highly effective salvage regimen in patients with refractory and relapsed acute myeloid leukemia of the poor risk: a final report of the Polish Adult Leukemia Group. Eur J Haematol. 2008;80(2):115-126.

10. Becker PS, Kantarjian HM, Appelbaum FR, et al. Clofarabine with high dose cytarabine and granulocyte colony-stimulating factor (G-CSF) priming for relapsed and refractory acute myeloid leukaemia. Br J Haematol. 2011;155(2):182-189.

11. Becker PS, Kantarjian HM, Appelbaum FR, et al. Retrospective comparison of clofarabine versus fludarabine in combination with high-dose cytarabine with or without granulocyte colony-stimurating factor as salvage therapies for acute myeloid leukemia. Haematological. 2013;98(1):114-118.

haematologica 2019; 104:e146 